OPEN ACCESS

Edited by:

Louis Bherer,

Université de Montréal, Canada

Reviewed by:

Katja Kornysheva,

University College London,

United Kingdom

Michael Thaut,

Colorado State University,

United States

*Correspondence: Valentin Bégel

valentin.begel@umontpellier.fr

Simone Dalla Bella

simone.dalla-bella@umontpellier.fr

Received: 15 October 2016 Accepted: 08 May 2017

Published: 29 May 2017

Citation:

Bégel V, Di Loreto I, Seilles A and Dalla Bella S (2017) Music Games: Potential Application and Considerations for Rhythmic Training.

Front. Hum. Neurosci. 11:273. doi: 10.3389/fnhum.2017.00273

\section{Music Games: Potential Application and Considerations for Rhythmic Training}

\author{
Valentin Bégel ${ }^{1,2 *}$, Ines Di Loreto ${ }^{3}$, Antoine Seilles ${ }^{2}$ and Simone Dalla Bella ${ }^{1,4,5,6 *}$ \\ ${ }^{1}$ Euromov Laboratory, University of Montpellier, Montpellier, France, ${ }^{2}$ NaturalPad, Montpellier, France, ${ }^{3}$ Homme, \\ Environnement et Technologies de l'Information, Université de Technologie de Troyes, Troyes, France, ${ }^{4}$ Institut Universitaire \\ de France, Paris, France, ${ }^{5}$ International Laboratory for Brain, Music and Sound Research (BRAMS), Montreal, QC, Canada, \\ ${ }^{6}$ Department of Cognitive Psychology, Wyższa Szkoła Finansów i Zarzadzanią w Warszawie (WSFiZ), Warsaw, Poland
}

Rhythmic skills are natural and widespread in the general population. The majority can track the beat of music and move along with it. These abilities are meaningful from a cognitive standpoint given their tight links with prominent motor and cognitive functions such as language and memory. When rhythmic skills are challenged by brain damage or neurodevelopmental disorders, remediation strategies based on rhythm can be considered. For example, rhythmic training can be used to improve motor performance (e.g., gait) as well as cognitive and language skills. Here, we review the games readily available in the market and assess whether they are well-suited for rhythmic training. Games that train rhythm skills may serve as useful tools for retraining motor and cognitive functions in patients with motor or neurodevelopmental disorders (e.g., Parkinson's disease, dyslexia, or ADHD). Our criteria were the peripheral used to capture and record the response, the type of response and the output measure. None of the existing games provides sufficient temporal precision in stimulus presentation and/or data acquisition. In addition, games do not train selectively rhythmic skills. Hence, the available music games, in their present form, are not satisfying for training rhythmic skills. Yet, some features such as the device used, the interface or the game scenario provide good indications for devising efficient training protocols. Guidelines are provided for devising serious music games targeting rhythmic training in the future.

Keywords: rhythm, serious game, rehabilitation, movement, training

\section{INTRODUCTION}

\section{Musical Rhythm as a Training Tool}

Humans display a natural tendency to move, spontaneously or deliberately, to the beat of rhythmic auditory stimuli, such as music (Repp, 2005; Repp and Su, 2013). This activity is widespread and is typically participatory. It manifests, for example, in dance, synchronized sport, and in group activities (e.g., waving together at a rock concert). Synchronization to a musical beat is sustained by a complex neuronal network, including perceptual regions (the superior temporal gyrus; Thaut, 2003; Chen et al., 2008a; Schwartze and Kotz, 2013), motor regions (e.g., the basal ganglia and the cerebellum; Grahn and Brett, 2007; Chen et al., 2008b; Grahn and Rowe, 2009), as well as sensorimotor integration areas (e.g., premotor cortex; Chen et al., 2006; Zatorre et al., 2007; Kornysheva and Schubotz, 2011). Disruption of these neuronal networks due to brain damage or neurodevelopmental disorder affects auditory-motor synchronization 
to a musical beat (Corriveau and Goswami, 2009; Bégel et al., 2017), as well as other functions such as speech. For example, difficulties encountered by individuals who stutter in speech production extend to non-verbal sensorimotor skills (Watkins et al., 2008; Falk et al., 2015). In speech production tasks, individual who stutter display reduced activity in brain regions that are also responsible for beat tracking and synchronization to a musical beat such as the basal ganglia (Civier et al., 2013), and the cerebellum (Brown et al., 2005).

Notably, tracking the beat does not require mandatorily an explicit motor response. In perceptual tasks, when detecting a deviation from the beat in an isochronous sequence (anisochrony detection; Ehrlé and Samson, 2005; Dalla Bella et al., 2017b), or detecting if a metronome is aligned or not to the beat (Beat Alignement Task, Iversen and Patel, 2008), the beat is extracted from the auditory signal to perform the judgment. Interestingly, beat extraction in the absence of an explicit motor response recruits motor regions of the brain, such as the basal ganglia (Grahn and Brett, 2007; Grahn and Rowe, 2009), or premotor cortex (Chen et al., 2008a,b). It is worth noting that processing sequences with an underlying beat engages partly separate mechanisms (beat-based timing), as compared to treating single durations (duration-based timing; Coull et al., 2011). The former rely on basal-ganglia-cortical mechanisms, while the latter are more associated to cerebellar-cortical pathways (Grube et al., 2010; Teki et al., 2011). For the purposes of this short review we focus in particular on the training of rhythmic skills engaging beat-based mechanisms.

Because mere listening to an auditory rhythm, for example conveyed via music, activates movement-related areas of the brain, training with rhythmic stimuli may be beneficial to (re)activate the motor system in the damaged and in the healthy brain. There are a few examples of the beneficial effects of rhythm on motor behavior. Rhythmic auditory stimulation can be used as a tool to retrain gait in Parkinson disease (e.g., for increasing speed and stride length; Thaut et al., 1996; Thaut and Abiru, 2010; Benoit et al., 2014; Dalla Bella et al., 2015, 2017a), to improve arm function in stroke (Thaut et al., 1997, 2002, 2007), or to enhance physical performance in sport (e.g., by reducing oxygen consumption in cycling; Hoffmann et al., 2012; Bardy et al., 2015). Positive effects of rhythmic training are not confined to motor behavior, but can extend to perception (Benoit et al., 2014; Dalla Bella et al., 2015). Stimulation using auditory rhythms shows promise also for training speech perception in children with Developmental Language Disorders (e.g., for syntax processing, Przybylski et al., 2013; Schön and Tillmann, 2015). In sum, previous studies point to beneficial effects of a rhythmic training protocol on movement and cognition. In addition, as rhythmic skills are linked to other cognitive abilities such as working memory and reading skills (Tierney and Kraus, 2013; Woodruff Carr et al., 2014), rhythmic training may foster improvements of more general cognitive abilities, which play a critical role in language learning and literacy (Schwartze and Kotz, 2013; Gordon et al., 2015; Kotz and Gunter, 2015). Ultimately, these beneficial effects of rhythmic training are likely to have positive consequences for health and well-being, such as promoting an active lifestyle, by reducing motor and cognitive decline in patient populations or reducing the need for healthcare services.

\section{Serious Games}

A great deal of work over the last two decades has been devoted to devise and promote games for training patients and for rehabilitation. This stream of research has been encouraged by low-cost and widespread new technologies offering unprecedented opportunities to implement training protocols. An increasing number of technologies are designed to improve health and well-being, from smartphone applications to control dietetics (Withings Wi-Fi Scales) to movement-based rehabilitation tools using motion capture (Zhou and $\mathrm{Hu}, 2008$; Weiss et al., 2009; Chang et al., 2011). Among them, video games provide a way to entertain people while targeting serious goals, such as the rehabilitation of impaired movement skills (e.g., Hammer and Planks, Di Loreto et al., 2013; Nintendo Wii games, Saposnik et al., 2010) or cognitive re-entrainment (e.g., RehaCom, Fernández et al., 2012) in neurological diseases (for a review and classification of serious games in health, see Rego et al., 2010). In particular, movement-based rehabilitation games exploiting motion capture devices such as the Wii or the Kinect are a promising way to use technology in the context of re-education (for a review see Webster and Celik, 2014). This method is referred to as "Exergaming". Note that video games for entertainment may also be used in a serious manner. For example, off-the-shelf video games are often used by physicians for therapeutic purposes, such as Nintendo Wii or Kinect games (Lange et al., 2009; Barry et al., 2014; Karahan et al., 2015). Exergaming has been proven as efficient in stroke (Webster and Celik, 2014), Parkinson's disease (Harris et al., 2015), as well as in healthy elderly adults (Sun and Lee, 2013).

During the last 5 years, studies have focused on the cognitive and neuronal underpinnings of the benefits linked to healthtargeted serious games (Connolly et al., 2012). On top of the physical and physiological benefits associated with serious games (e.g., via dedicated physiotherapeutic exercises), the effects of this type of training extend to cognition. Cognitive functions such as language and memory can also be enhanced by serious games, an effect which is likely to be accompanied by plastic changes of the brain. For example, structural brain changes associated with learning have been observed due to the use of videogames (Anguera et al., 2013). These promising results indicate that implementing training protocols via serious games may be particularly valuable for enhancing brain functions as well as for therapy and rehabilitation.

In summary, serious games and rhythmic stimulation are promising tools that can be exploited to improve or retrain movement and cognition. We propose that a training of rhythm skills implemented in a serious game would be a means to set up training protocols which may serve rehabilitation of different patient populations. The aim of this review article is to provide an overview of the existing rhythm games and to assess whether they could be well-suited for training purposes. We conducted a survey in which we used criteria such as the precision of the recorded response or the modality of the stimuli provided to evaluate the benefits and limitations of each game. 


\section{LIMITS AND ADVANTAGES OF THE EXISTING RHYTHM GAMES}

To the best of our knowledge, only one music-based training program that uses a game setting has been successfully devised for arm rehabilitation in stroke patients (Friedman et al., 2011, 2014). However, this protocol is not training rhythmic skills per se but is rather a music-based adaptation of a standard rehabilitation protocol (i.e., conventional tabletop exercises therapy; Dickstein et al., 1986). To examine whether existing games involving rhythm conveyed by auditory stimuli could be potentially used as training tools, we selected games based on the following inclusion criteria. First, the game has to focus on rhythmic skills. The player must be instructed to synchronize movement (or voice) with stimuli (auditory or visual) which can be predicted on the basis of their temporal structure (i.e., the underlying beat). To our knowledge, no rhythm games currently on the market use purely perceptual tasks, in which the player's task is to judge the rhythmic features of music. All the games presented below involve movement synchronized to auditory or visual cues. Second, the game device must record the temporal precision of the player's responses. The scores, levels, difficulty and feedback given to the player must depend on her/his temporal precision in performing the movement. Once the games were selected, they were categorized by: (a) the peripheral used to capture and record the response; (b) the type of response that is recorded; and (c) the output. The peripheral is important to judge if the game is readily usable for training (e.g., in a clinical context). In addition, note that most of the studies in cognitive psychology of rhythm use finger tapping, since this is a simple and objective way to study rhythmic skills (Repp, 2005; Repp and Su, 2013), but other reponses are possible (e.g., full-body motion). Finally, the output is relevant as it indicates whether the games provide a feedback (an outcome measure or score) on the precision of the performance reflecting a participant's rhythmic skills. These categories are helpful for evaluating the therapeutic potential of each game. For example, a game requiring finger tapping is likely to have a different effect on behavior than a game requiring full body motion, such as dance.

Twenty-seven games on a variety of devices (Wii, PlayStation, PC, Tablet/Smartphone, Xbox, Gameboy) fulfilling the aforementioned criteria were considered for the analysis (see Table 1). These games were classified in four categories as indicated below.

\section{Games that Involve Full Body Movements Recorded via an External Interface (e.g., Kinect, Wii)}

Here we refer mostly to dance games (e.g., Just Dance). These games have interesting applications in physiotherapy for patients with spinal cord injury, traumatic brain injury and stroke (Lange et al., 2009). They focus more on physical exercise and activity than on rhythm per se. Indeed, the ability of these games to record and score the rhythmic precision of the player is rather poor. Because these games focus on discrete movements/actions instead of repeated movements (i.e., rhythmic) they cannot be used for delivering specific training of rhythmic skills. For example, Just Dance consists in reproducing movements that are illustrated through images displayed on the screen. The player's score depends on the precision of the movements as compared to a model action sequence. The player has to execute the movements in a given temporal window. Yet, the task is not purely rhythmic and synchronization to the musical beat is not recorded. In spite of the fact that these games do not measure rhythmic skills per se, they provide a motivating setting to perform dance while monitoring the player's movements. Adding a rhythm component to some of these games, as in the case of dance, may be a valuable strategy to translate them into a training program.

\section{Games that Involve Rhythmic Finger Tapping on a Tablet}

An example of these games is Beat Sneak Bandit. Here, the player has to tap precisely to the beat in order to make the character progress, avoid the enemies and so forth. This kind of feature is used in serious games dedicated to learning, such as Rhythm Cat, designed to learn music rhythm notation. For the purposes of training rhythmic skills, one major drawback of these games is that the timing precision of the software is very poor. The time window in which a response is considered as good is very wide (i.e., up to several hundreds of milliseconds) and the temporal variability of the recording is high. In addition, no feedback on the rhythmic performance of the player is provided.

\section{Computer or Console Games that Involve Finger Tapping on Keys}

These games can be played on a keyboard, using a joystick, or on special devices. One of the most famous is Guitar Hero. In this game the player plays on a guitar replica with five keys, and has to push the keys in correspondence of images presented on a screen. Rhythm precision of the responses is recorded and used to compute a performance score. The response must appear in a specific temporal window to be considered as good. The same concept is used in many PC games, but keyboards key (e.g., arrows) are used instead of guitar replica. As in the case of tablet games, the main weakness of these games is their low temporal precision in recording rhythmic performance (around $100 \mathrm{~ms}$ in Guitar Hero). Nevertheless, these games are interesting as they represent a good starting point to develop serious-game applications aimed at training rhythmic skills.

\section{Console Games Involving Singing}

In these games, the player is asked to sing in synchrony with the music. This is not a rhythmic task per se, but the performance involves a rhythmic component. As in classical karaoke, lyrics are presented on the screen. In this case, a feedback (score) is provided to the player while she/he sings and a final global score is given at the end of the performance, including temporal precision (the response must appear in a given temporal window to be considered as good) but also pitch precision. Here, the potential benefit for health rests upon the fact that singing is 
TABLE 1 | List of the reviewed rhythm-based games.

\begin{tabular}{|c|c|c|c|}
\hline Game & Peripheral & Type of response recorded & Output \\
\hline Dance revolution/dancing stage & Dance pad (PS2, PC) & Impacts of feet (PS2)/fingers (PC) & Incrementing score \\
\hline Donkey konga & Bongos & Impacts of hands & Incrementing score \\
\hline Dancing with the star & $\begin{array}{l}\text { Wiimote, Nunchuk (Wii), keyboard } \\
\text { (PC) }\end{array}$ & $\begin{array}{l}\text { Hands movement (Wiimote), Key } \\
\text { tapping (PC) }\end{array}$ & Incrementing score \\
\hline DJ hero & $\begin{array}{l}\text { Turntable replica (Wii, PS } 2 \text { and } 3 \text {, } \\
\text { Xbox } 360 \text { ) }\end{array}$ & $\begin{array}{l}\text { Hands and fingers movement on } \\
\text { the Turntable }\end{array}$ & Incrementing score \\
\hline Everyone sing & Microphone (Wii, PS 3, Xbox 360) & Voice & Incrementing score \\
\hline Guitar hero & $\begin{array}{l}\text { Guitar replica, joystick (Wii, PS 3, } \\
\text { Xbox 360), keyboard (PC), screen } \\
\text { (tablet, Androîd) }\end{array}$ & $\begin{array}{l}\text { Left-hand key tapping, right-hand } \\
\text { key moving up and down (Wii, PS3, } \\
\text { Xbox 360), screen tapping (tablet), } \\
\text { joystick button pressing (Wiimote, } \\
\text { pS3, Xbox 360) }\end{array}$ & Incrementing score \\
\hline Just dance & $\begin{array}{l}\text { Wiimote (Wii), PS camera, PS move } \\
\text { (PS4, PS3), Kinect (Xbox 360, Xbox } \\
\text { one) }\end{array}$ & $\begin{array}{l}\text { Hand movement (Wiimote), all-body } \\
\text { movement (PS move, PS camera, } \\
\text { Kinect) }\end{array}$ & Incrementing score \\
\hline Rhythm paradise (USA: Rhythm Heaven Fever) & Nintendo DS, Wiimote & $\begin{array}{l}\text { Finger tapping on the screen, hand } \\
\text { movement (stylus; DS), key tapping } \\
\text { (Wii) }\end{array}$ & Incrementing score \\
\hline Rock band & $\begin{array}{l}\text { Guitar, Drums replica, Microphone } \\
\text { (Wii, Xbox 360, PS3), Tactile screen } \\
\text { (Iphone, Ipod Touch), Nintendo DS, } \\
\text { PSP }\end{array}$ & $\begin{array}{l}\text { Left-hand key tapping, right-hand } \\
\text { key moving up and down } \\
\text { (mediator-like), feet impact (bass } \\
\text { drum), drumsticks impact (Wii, PS3, } \\
\text { Xbox 360, Nintendo DS, PSP), } \\
\text { screen tapping (Iphone, Ipod), } \\
\text { joystick button pressing (Wiimote, } \\
\text { pS3, Xbox 360), voice (microphone) }\end{array}$ & Incrementing score \\
\hline 140 & Keyboard (PC) & Key pressing & Progression in a level \\
\hline Osu & Mouse (PC) & Key pressing & Incrementing score \\
\hline Beatmania & $\begin{array}{l}\text { Turntable replica (Arcade, PS1, } \\
\text { PS2), Nintendo gameboy }\end{array}$ & $\begin{array}{l}\text { Hands and fingers movement on } \\
\text { the Turntable (Arcade, PS1, PS2), } \\
\text { key pressing (Gameboy color) }\end{array}$ & Incrementing score \\
\hline Patapon & PSP & Key tapping & Progression in a level \\
\hline Rhythm cat & Tablet, Smartphone & Screen tapping, holding, swiping & Incrementing score \\
\hline Groove coaster zero & Tablet, Smartphone & Screen tapping, holding, swiping & Incrementing score \\
\hline lgobeat & Tablet, Smartphone & Screen tapping, holding, swiping & Incrementing score \\
\hline Beat brite & Tablet, Smartphone & Screen tapping, holding, swiping & Incrementing score \\
\hline Online PC games & Keyboard & Screen tapping & $\begin{array}{l}\text { Progression in a level/Incrementing } \\
\text { score }\end{array}$ \\
\hline
\end{tabular}

The last row concerns online PC games (available at www.musicgames.co/games-by-category/rhythm-games/) having similar characteristics.

a good way to restore speech abilities (e.g., fluency) in aphasia following stroke (for example, see Norton et al., 2009).

Even though some of the aforementioned games present good ground for training rhythmic skills, their main drawback is that their temporal precision when recording movement relative to the beat is rather poor. Thus, the output measures provided by these games are insufficient to isolate rhythmic features of the performance (e.g., variability of the motor performance, precision of the synchronization with the beat, etc.). Moreover, in none of these games the rhythmic complexity of musical stimuli has been manipulated. Difficulty is manipulated only through the amount of responses required during the game (e.g., number of visual tags which the player has to react to) which is not a rhythmical feature. For example, using music with various degrees of beat saliency would allow introducing rhythm-based difficulty levels in the game. This has the advantage that rhythms with increased complexity could be presented progressively throughout the game, thus potentially leading to improved beat-tracking skills.

\section{CONCLUSION}

We reviewed 27 rhythm-based games already in the market that could be used in a rhythmic training protocol. Unfortunately, based on our criteria, none of the aforementioned games is satisfying for this purpose. First, in most of the games, the task consists in reacting to visual stimulations while music is presented. Thus rhythmic skills are not selectively trained. Second, the number of stimuli, instead of the rhythmic characteristics of the music, is varied to change the difficulty of the game. Third, in spite of the fact that the regularity of rhythmic patterns can influence the performance in the game, the response provided by the player is not targeted at the rhythmic aspects of the stimuli. For example, the player touches the screen at the right moment to catch objects or makes full-body movements to imitate model-actions in dance games. In addition, note that the reviewed games do not offer opportunities for controlled functional movement training. For example, none of them provide a guidance to achieve desired movement trajectories. This problem may be 
overcome in the future by providing relevant feedback when the player approaches optimal movement trajectories (e.g., via sonification, Effenberg et al., 2016). The tasks implemented in these games are vaguely reminiscent of implicit timing tasks (Lee, 1976; Zelaznik et al., 2002; Coull and Nobre, 2008). Explicit and implicit timing have been treated as relatively independent processes in cognitive neuroscience (Zelaznik et al., 2002; Coull and Nobre, 2008; Coull et al., 2011). The former is associated with tasks requiring voluntary motor production (e.g., synchronized tapping tasks; Repp, 2005; Repp and Su, 2013) or overt estimation of stimulus duration (e.g., duration discrimination; Grondin, 1993). In contrast, implicit timing is tested with tasks unrelated to timing (e.g., avoiding a vehicle when crossing the road), but in which temporal prediction affects the performance (judging the time before the vehicle reaches us; Lee, 1976; for more details, see Nobre et al., 2007; Coull and Nobre, 2008; Coull, 2009). In particular, temporal prediction fostered by a regular temporal pattern (e.g., a metronome) of sensory stimuli improves performance in non-temporal tasks (e.g., working memory, Cutanda et al., 2015; pitch judgment, Jones et al., 2002; language judgments, Przybylski et al., 2013).

Despite the available music games are not explicitely targeted at rhythmic training, they may still foster training timing implicitly, in combination with other more explicit processes (e.g., focusing on spatial and pitch accuracy). There is evidence that the implicit dimension of timing may be more robust than explicit timing, for example in beat deafness (Bégel et al., 2017). It is possible that participants with timing disorders (e.g., Parkinson's disease or developmental stuttering; Grahn and Brett, 2009; Falk et al., 2015) may still be able to capitalize on partly spared implicit timing functions to re-learn rhythmic skills via a training program. Note, however, that so far beneficial effects of rhythm-based training protocols typically made use of explicit timing tasks (e.g., walking with an auditory rhythm; e.g., Lim et al., 2005; Spaulding et al., 2013; Benoit et al., 2014). This may suggest that tasks which recruit explicit timing mechanisms may be a particularly good candidate to build a successful protocol for rhythmic training. In only one of the reviewed games (Beat Sneak Bandit), the goal was to tap to the beat of music, which is an explicit timing task. In sum, almost all of the reviewed games do not require participants to perform explicitly rhythmic tasks. Yet, they are likely to engage implicit timing mechanisms. Whether training rhythm implicitely in the context of a music game can lead to positive effects comparable to those found with explicit rhythmic tasks deserves further enquiry.

\section{REFERENCES}

Anguera, J. A., Boccanfuso, J., Rintoul, J. L., Al-Hashimi, O., Faraji, F., Janowich, J., et al. (2013). Video game training enhances cognitive control in older adults. Nature 501, 97-101. doi: 10.1038/nature12486

Bardy, B. G., Hoffmann, C. P., Moens, B., Leman, M., and Dalla Bella, S. (2015). Sound-induced stabilization of breathing and moving. Ann. N Y Acad. Sci. 1337, 94-100. doi: $10.1111 /$ nyas. 12650
In summary, the games currently on the market, albeit they are not optimal for rhythmic training, provide at least interesting ideas that might pave the ground to devise successful training programs. Games on portable devices (e.g., tablets or smartphones) using tapping to the beat provide the simplest solution to implement a rhythm training protocol. They are low-cost while offering a motivating and user-friendly environment to train rhythmic skills with a playful interface. Although this solution has some potential, there are two problems. The precision of movement recording relative to the beat, and the ensuing measures of rhythm precision, need significant improvement. To deal with these issues, methods used to analyze synchronization to the beat in the neurosciences of rhythm (Kirschner and Tomasello, 2009; Pecenka and Keller, 2009; Woodruff Carr et al., 2014; Dalla Bella et al., 2017b) should be applied to games designed for rhythm training. In addition, estimations of timekeeper and motor implementation variance (e.g., based on tapping performance) might allow to refine the feedback on the performance (e.g., Schulze and Vorberg, 2002; for a review see, Wing, 2002). This will ensure that a precise feedback on the rhythmic performance can be provided and that the stimuli and game progression can be tailored to individual learning curves. Moreover, to ensure that the training program specifically targets rhythmic skills, stimulus (or response) will have to be varied in terms of rhythmic difficulty. This can be achieved, for example, by selecting musical excerpts based on their rhythmic complexity. Using stimuli with increasing difficulty in beat tracking (e.g., with a less salient beat) throughout the game might allow to progressively fine tune the player's rhythmic skills. These guidelines should be taken into account in the future to devise efficient protocols for training rhythmic skills via serious music games.

\section{AUTHOR CONTRIBUTIONS}

VB and SDB conceived the study; VB conducted the survey; $\mathrm{VB}$, IDL and AS contributed to data analysis; all authors contributed to the writing of the manuscript.

\section{FUNDING}

Junior Grant from the Institut Universitaire de France to SDB.

\section{ACKNOWLEDGMENTS}

We wish to thank two reviewers for their insightful comments on a first draft of the manuscript.

Barry, G., Galna, B., and Rochester, L. (2014). The role of exergaming in Parkinson's disease rehabilitation: a systematic review of the evidence. J. Neuroeng. Rehabil. 11:33. doi: 10.1186/1743-000311-33

Bégel, V., Benoit, C.-E., Correa, A., Cutanda, D., Kotz, S. A., and Dalla Bella, S. (2017). "Lost in time" but still moving to the beat. Neuropsychologia 94, 129-138. doi: 10.1016/j.neuropsychologia.2016. 11.022 
Benoit, C.-E., Dalla Bella, S., Farrugia, N., Obrig, H., Mainka, S., and Kotz, S. A. (2014). Musically cued gait-graining improves both perceptual and motor timing in Parkinson's disease. Front. Hum. Neurosci. 8:494. doi: 10.3389/fnhum.2014.00494

Brown, S., Ingham, R. J., Ingham, J. C., Laird, A. R., and Fox, P. T. (2005). Stuttered and fluent speech production: an ALE meta-analysis of functional neuroimaging studies. Hum. Brain Mapp. 25, 105-117. doi: 10.1002/hbm. 20140

Chang, Y. J., Chen, S. F., and Huang, J. D. (2011). A kinect-based system for physical rehabilitation: a pilot study for young adults with motor disabilities. Res. Dev. Disabil. 32, 2566-2570. doi: 10.1016/j.ridd.2011. 07.002

Chen, J. L., Zatorre, R. J., and Penhune, V. B. (2006). Interactions between auditory and dorsal premotor cortex during synchronization to musical rhythms. Neuroimage 32, 1771-1781. doi: 10.1016/j.neuroimage.2006.04.207

Chen, J. L., Penhune, V. B., and Zatorre, R. J. (2008a). Moving on time: brain network for auditory-motor synchronization is modulated by rhythm complexity and musical training. J. Cogn. Neurosci. 20, 226-239. doi: 10.1162/jocn.2008.20018

Chen, J. L., Penhune, V. B., and Zatorre, R. J. (2008b). Listening to musical rhythms recruits motor regions of the brain. Cereb. Cortex 18, 2844-2854. doi: 10.1093/cercor/bhn042

Civier, O., Bullock, D., Max, L., and Guenther, F. H. (2013). Computational modeling of stuttering caused by impairments in a basal ganglia thalamocortical circuit involved in syllable selection and initiation. Brain Lang. 126, 263-278. doi: 10.1016/j.bandl.2013.05.016

Connolly, T. M., Boyle, E. A., MacArthur, E., Hainey, T., and Boyle, J. M. (2012). A systematic literature review of empirical evidence on computer games and serious games. Comput. Educ. 59, 661-686. doi: 10.1016/j.compedu.2012. 03.004

Corriveau, K. H., and Goswami, U. (2009). Rhythmic motor entrainment in children with speech and language impairments: tapping to the beat. Cortex 45, 119-130. doi: 10.1016/j.cortex.2007.09.008.

Coull, J. T. (2009). Neural substrates of mounting temporal expectation. PLoS Biol. 7:e1000166. doi: 10.1371/journal.pbio.1000166

Coull, J. T., Cheng, R. K., and Meck, W. H. (2011). Neuroanatomical and neurochemical substrates of timing. Neuropsychopharmacology 36, 3-25. doi: $10.1038 /$ npp. 2010.113

Coull, J. T., and Nobre, A. C. (2008). Dissociating explicit timing from temporal expectation with fMRI. Curr. Opin. Neurobiol. 18, 137-144. doi: 10.1016/j.conb. 2008.07.011

Cutanda, D., Correa, Á., and Sanabria, D. (2015). Auditory temporal preparation induced by rhythmic cues during concurrent auditory working memory tasks. J. Exp. Psychol. Hum. Percept. Perform. 41, 790-797. doi: 10.1037/a00 39167

Dalla Bella, S., Benoit, C.-E., Farrugia, N., Schwartze, M., and Kotz, S. A. (2015). Effects of musically cued gait training in Parkinson's disease: beyond a motor benefit. Ann. N Y Acad. Sci. 1337, 77-85. doi: 10.1111/nyas.12651

Dalla Bella, S., Benoit, C.-E., Farrugia, N., Keller, P. E., Obrig, H., Mainka, S., et al. (2017a). Gait improvement via rhythmic stimulation in Parkinson's disease is linked to rhythmic skills. Sci. Rep. 7:42005. doi: 10.1038/srep42005

Dalla Bella, S., Farrugia, N., Benoit, C. -E., Begel, V., Verga, L., Harding, E., et al. (2017b). BAASTA: battery for the assessment of auditory sensorimotor and timing abilities. Behav. Res. Methods 49, 1128-1145. doi: 10.3758/s13428-0160773-6

Di Loreto, I., Lange, B., Seilles, A., Andary, S., and Dyce, W. (2013). “Game design for all: the example of hammer and planks a game for rehabilitation purposes or a game for all?" in 4th International Conference on Serious Games Development and Applications (SGDA), LNCS 323 8101, (Norway), 70-75.

Dickstein, R., Hocherman, S., Pillar, T., and Shaham, R. (1986). Stroke rehabilitation. three exercise therapy approaches. Phys. Ther. 66, 1233-1238.

Effenberg, A. O., Fehse, U., Schmitz, G., Krueger, B., and Mechling, H. (2016). Movement sonification: effects on motor learning beyond rhythmic adjustments. Front. Neurosci. 10:219. doi: 10.3389/fnins.2016. 00219

Ehrlé, N., and Samson, S. (2005). Auditory discrimination of anisochrony: influence of the tempo and musical backgrounds of listeners. Brain Cogn. 58, 133-147. doi: 10.1016/j.bandc.2004.09.014
Falk, S., Müller, T., and Dalla Bella, S. (2015). Non-verbal sensorimotor timing deficits in children and adolescents who stutter. Front. Psychol. 6:847. doi: 10.3389/fpsyg.2015.00847

Fernández, E., Bringas, M. L., Salazar, S., Rodríguez, D., García, M. E., and Torres, M. (2012). Clinical impact of RehaCom software for cognitive rehabilitation of patients with acquired brain injury. MEDICC Rev. 14, 32-35. doi: 10.1590/s1555-79602012000400007

Friedman, N., Chan, V., Reinkensmeyer, A. N., Beroukhim, A., Zambrano, G. J., Bachman, M., et al. (2014). Retraining and assessing hand movement after stroke using the musicglove: comparison with conventional hand therapy and isometric grip training. J. Neuroeng. Rehabil. 11:76. doi: 10.1186/1743-00 03-11-76

Friedman, N., Reinkensmeyer, D., and Bachman, M. (2011). A real-time interactive MIDI glove for domicile stroke rehabilitation. Lect. Notes Comp. Sci. 6764, 151-158. doi: 10.1007/978-3-642-21619-0_20

Gordon, R. L., Shivers, C. M., Wieland, E. A., Kotz, S. A., Yoder, P. J., and McAuley, J. D. (2015). Musical rhythm discrimination explains individual differences in grammar skills in children. Dev. Sci. 18, 635-644. doi: $10.1111 /$ desc. 12230

Grahn, J. A., and Brett, M. (2007). Rhythm and beat perception in motor areas of the brain. J. Cogn. Neurosci. 19, 893-906. doi: 10.1162/jocn.2007.19.5.893

Grahn, J. A., and Brett, M. (2009). Impairment of beat-based rhythm discrimination in Parkinson's disease. Cortex 45, 54-61. doi: 10.1016/j.cortex. 2008.01.005

Grahn, J. A., and Rowe, J. B. (2009). Feeling the beat: premotor and striatal interactions in musicians and nonmusicians during beat perception. J. Neurosci. 29, 7540-7548. doi: 10.1523/JNEUROSCI.2018-08.2009

Grondin, S. (1993). Duration discrimination of empty and filled intervals marked by auditory and visual signals. Percept. Psychophys. 54, 383-394. doi: 10.3758/bf03205274

Grube, M., Cooper, F. E., Chinnery, P. F., and Griffiths, T. D. (2010). Dissociation of duration-based and beat-based auditory timing in cerebellar degeneration. Proc. Natl. Acad. Sci. U S A 107, 11597-11601. doi: 10.1073/pnas.0910473107

Harris, D. M., Rantalainen, T., Muthalib, M., Johnson, L., and Teo, W.-P. (2015). Exergaming as a viable therapeutic tool to improve static and dynamic balance among older adults and people with idiopathic Parkinson's disease: a systematic review and meta-analysis. Front. Aging Neurosci. 7:167. doi: 10.3389/fnagi.2015. 00167

Hoffmann, C. P., Torregrosa, G., and Bardy, B. G. (2012). Sound stabilizes locomotor-respiratory coupling and reduces energy cost. PLoS One 7:e45206. doi: 10.1371/journal.pone.0045206

Iversen, J. R., and Patel, A. D. (2008). "The beat alignment test (BAT): surveying beat processing abilities in the general population," in Proceedings of the 10th International Conference on Music Perception \& Cognition (ICMPC10), August 2008, Sapporo, Japan, ed. K. Miyazaki (Adelaide: Causal Productions), 465-468.

Jones, M. R., Moynihan, H., MacKenzie, N., and Puente, J. (2002). Temporal aspects of stimulus-driven attending in dynamic arrays. Psychol. Sci. 13, 313-319. doi: 10.1111/1467-9280.00458

Karahan, A. Y., Tok, F., Tașkin, H., Küçüksaraç, S., Basaran, A., and Yildirim, P. (2015). Effects of exergames on balance, functional mobility and quality of life of geriatrics versus home exercise programme: randomized controlled study. Cent. Eur. J. Public Health 23, S14-S18. doi: 10.21101/cejph.a4081

Kirschner, S., and Tomasello, M. (2009). Joint drumming: social context facilitates synchronization in preschool children. J. Exp. Child Psychol. 102, 299-314. doi: 10.1016/j.jecp.2008.07.005

Kornysheva, K., and Schubotz, R. I. (2011). Impairment of auditory-motor timing and compensatory reorganization after ventral premotor cortex stimulation. PLoS One 6:e21421. doi: 10.1371/journal.pone.0021421

Kotz, S. A., and Gunter, T. C. (2015). Can rhythmic auditory cuing remediate language-related deficits in Parkinson's disease? Ann. N Y Acad. Sci. 1337, 62-68. doi: 10.1111/nyas.12657

Lange, B., Flynn, S., and Rizzo, A. (2009). Initial usability assessment of off-theshelf video game consoles for clinical game-based motor rehabilitation. Phys. Ther. Rev. 14, 355-363. doi: 10.1179/108331909x12488667117258

Lee, D. N. (1976). A theory of visual control of braking based on information about time-to-collision. Perception 5, 437-459. doi: 10.1068/p050437

Lim, I., Van Wegen, E., de Goede, C., Deutekom, M., Nieuwboer, A., Willems, A., et al. (2005). Effects of external rhythmical cueing on gait in patients 
with Parkinson's disease: a systematic review. Clin. Rehabil. 19, 695-713. doi: 10.1191/0269215505cr906oa

Nobre, A. C., Correa, A., and Coull, J. T. (2007). The hazards of time. Curr. Opin. Neurobiol. 17, 465-470. doi: 10.1016/j.conb.2007.07.006

Norton, A., Zipse, L., Marchina, S., and Schlaug, G. (2009). Melodic intonation therapy: shared insights on how it is done and why it might help. Ann. N Y Acad. Sci. 1169, 431-436. doi: 10.1111/j.1749-6632.2009.04859.x

Pecenka, N., and Keller, P. E. (2009). Auditory pitch imagery and its relationship to musical synchronization. Ann. N Y Acad. Sci. 1169, 282-286. doi: 10.1111/j. 1749-6632.2009.04785.x

Przybylski, L., Bedoin, N., Krifi-Papoz, S., Herbillon, V., Roch, D., Léculier, L., et al. (2013). Rhythmic auditory stimulation influences syntactic processing in children with developmental language disorders. Neuropsychology 27, 121-131. doi: $10.1037 / \mathrm{a} 0031277$

Rego, P., Moreira, P., and Reis, L. (2010). "Serious games for rehabilitiation: a survey and classification towards a taxonomy," in 5th Iberian Conference on Information Systems and Technologies (CISTI), (Santiago de Compos tela), $1-6$.

Repp, B. H. (2005). Sensorimotor synchronization: a review of the tapping literature. Psychon. Bull. Rev. 12, 969-992. doi: 10.3758/bf03206433

Repp, B. H., and Su, Y.-H. (2013). Sensorimotor synchronization: a review of recent research (2006-2012). Psychon. Bull. Rev. 20, 403-452. doi: 10.3758/s13423-012-0371-2

Saposnik, G., Teasell, R., Mamdani, M., Hall, J., McIlroy, W., Cheung, D., et al. (2010). Effectiveness of virtual reality using Wii gaming technology in stroke rehabilitation: a pilot randomized clinical trial and proof of principle. Stroke 41, 1477-1484. doi: 10.1161/STROKEAHA.110.584979

Schön, D., and Tillmann, B. (2015). Short- and long-term rhythmic interventions: perspectives for language rehabilitation. Ann. N Y Acad. Sci. 1337, 32-39. doi: $10.1111 /$ nyas. 12635

Schulze, H. H., and Vorberg, D. (2002). Linear phase correction models for synchronization: parameter identification and estimation of parameters. Brain Cogn. 48, 80-97. doi: 10.1006/brcg.2001.1305

Schwartze, M., and Kotz, S. A. (2013). A dual-pathway neural architecture for specific temporal prediction. Neurosci. Biobehav. Rev. 37, 2587-2596. doi: 10.1016/j.neubiorev.2013.08.005

Spaulding, S. J., Barber, B., Colby, M., Cormack, B., Mick, T., and Jenkins, M. E. (2013). Cueing and gait improvement among people with Parkinson's disease: a meta-analysis. Arch. Phys. Med. Rehabil. 94, 562-570. doi: 10.1016/j.apmr. 2012.10.026

Sun, T. L., and Lee, C. H. (2013). An impact study of the design of exergaming parameters on body intensity from objective and gameplay-based player experience perspectives, based on balance training exergame. PLoS One 8:e69471. doi: 10.1371/journal.pone.0069471

Teki, S., Grube, M., Kumar, S., and Griffiths, T. D. (2011). Distinct neural substrates of duration-based and beat-based auditory timing. J. Neurosci. 31, 3805-3812. doi: 10.1523/JNEUROSCI.5561-10.2011

Thaut, M. H. (2003). Neural basis of rhythmic timing networks in the human brain. Ann. N Y Acad. Sci. 999, 364-373. doi: 10.1196/annals.1284.044

Thaut, M. H., and Abiru, M. (2010). Rhythmic auditory stimulation in rehabilitation of movement disorders: a review of current research. Music Percept. 27, 263-269. doi: 10.1525/mp.2010.27.4.263

Thaut, M. H., Kenyon, G. P., Hurt, C. P., McIntosh, G. C., and Hoemberg, V. (2002). Kinematic optimization of spatiotemporal patterns in paretic arm training with stroke patients. Neuropsychologia 40, 1073-1081. doi: 10.1016/s0028-3932(01)00141-5

Thaut, M. H., Leins, A. K., Rice, R. R., Argstatter, H., Kenyon, G. P., McIntosh, G. C., et al. (2007). Rhythmic auditor y stimulation improves gait more than NDT/Bobath training in near-ambulatory patients early poststroke: a single-blind, randomized trial. Neurorehabil. Neural Repair 21, 455-459. doi: $10.1177 / 1545968307300523$

Thaut, M. H., McIntosh, G. C., Rice, R. R., Miller, R. A., Rathbun, J., and Brault, J. M. (1996). Rhythmic auditory stimulation in gait training for Parkinson's disease patients. Mov. Disord. 11, 193-200. doi: 10.1002/mds. 870110213

Thaut, M. H., McIntosh, G. C., and Rice, R. R. (1997). Rhythmic facilitation of gait training in hemiparetic stroke rehabilitation. J. Neurol. Sci. 151, 207-212. doi: $10.1016 / \mathrm{s} 0022-510 x(97) 00146-9$

Tierney, A. T., and Kraus, N. (2013). The ability to tap to a beat relates to cognitive, linguistic and perceptual skills. Brain Lang. 124, 225-231. doi: 10.1016/j.bandl. 2012.12.014

Watkins, K. E., Smith, S. M., Davis, S., and Howell, P. (2008). Structural and functional abnormalities of the motor system in developmental stuttering. Brain 131, 50-59. doi: 10.1093/brain/awm241

Webster, D., and Celik, O. (2014). Systematic review of kinect applications in elderly care and stroke rehabilitation. J. Neuroeng. Rehabil. 11:108. doi: 10.1186/1743-0003-11-108

Weiss, P. L., Sveistrup, H., Rand, D., and Kizony, R. (2009). Video capture virtual reality: a decade of rehabilitation assessment and intervention. Phys. Ther. Rev. 14, 307-321. doi: 10.1179/108331909x12488667117339

Wing, A. M. (2002). Voluntary timing and brain function: an information processing approach. Brain Cogn. 48, 7-30. doi: 10.1006/brcg.20 01.1301

Woodruff Carr, K., White-Schwoch, T., Tierney, A. T., Strait, D. L., and Kraus, N. (2014). Beat synchronization predicts neural speech encoding and reading readiness in preschoolers. Proc. Natl. Acad. Sci. U S A 111, 14559-14564. doi: 10.1073/pnas.1406219111

Zatorre, R. J., Chen, J. L., and Penhune, V. B. (2007). When the brain plays music: auditory-motor interactions in music perception and production. Nat. Rev. Neurosci. 8, 547-558. doi: 10.1038/nrn2152

Zelaznik, H. N., Spencer, R., and Ivry, R. B. (2002). Dissociation of explicit and implicit timing in repetitive tapping and drawing movements. J. Exp. Psychol. Hum. Percept. Perform. 28, 575-588. doi: 10.1037/0096-1523.28.3.575

Zhou, H., and $\mathrm{Hu}, \mathrm{H}$. (2008). Human motion tracking for rehabilitation-A survey. Biomed. Signal Process. Control 3, 1-18. doi: 10.1016/j.bspc.2007. 09.001

Conflict of Interest Statement: The authors declare that the research was conducted in the absence of any commercial or financial relationships that could be construed as a potential conflict of interest.

Copyright (c) 2017 Bégel, Di Loreto, Seilles and Dalla Bella. This is an open-access article distributed under the terms of the Creative Commons Attribution License (CC BY). The use, distribution or reproduction in other forums is permitted, provided the original author(s) or licensor are credited and that the original publication in this journal is cited, in accordance with accepted academic practice. No use, distribution or reproduction is permitted which does not comply with these terms. 